\title{
CORRECTION
}

\section{Correction: Steady-state mycophenolate mofetil pharmacokinetic parameters enable prediction of systemic lupus erythematosus clinical flares: an observational cohort study}

\author{
Sarah Djabarouti*1, Dominique Breilh1', Pierre Duffau², Estibaliz Lazaro², Carine Greib², Olivier Caubet², \\ Marie-Claude Saux', Jean-Luc Pellegrin² and Jean-François Viallard²
}

See related research by Djabarouti et al., http://arthritis-research.com/content/12/6/R217

After publication of our recent article [1], we noticed an error in the unit for the area under the timeconcentration curve between 0 and 12 hours $\left(\mathrm{AUC}_{0-12 \mathrm{~h}}\right)$. Throughout the article and table 1 the unit should be $\mathrm{mg} \cdot \mathrm{h} / \mathrm{L}$ (mg multiplied by hour per L) not mg/hour/L .

The correct table is given here as Table 1.

Competing interests

The authors declare that they have no competing interests.

\section{Author details}

'Laboratoire de Pharmacocinétique et Pharmacie Clinique EA2968, Université Victor Segalen Bordeaux 2, Hôpital Haut-Lévêque, CHU de Bordeaux, Avenue de Magellan, 33604 Pessac Cedex, France. ${ }^{2}$ Service de Médecine Interne et des Maladies Infectieuses, Université Victor Segalen Bordeaux 2, Hôpital HautLévêque, CHU de Bordeaux, Avenue de Magellan, 33604 Pessac Cedex, France.
Published: 3 March 2011

\section{Reference}

1. Djabarouti S, Breilh D, Duffau P, Lazaro E, Greib C, Caubet O, Saux MC, Pellegrin JL, Viallard JF: Steady-state mycophenolate mofetil pharmacokinetic parameters enable prediction of systemic lupus erythematosus clinical flares: an observational cohort study. Arthritis Res Ther 2010, 12:R217

doi:10.1186/ar3265

Cite this article as: Djabarouti S, et al.: Correction: Steady-state

mycophenolate mofetil pharmacokinetic parameters enable prediction of systemic lupus erythematosus clinical flares: an observational cohort study. Arthritis Research \& Therapy 2011, 13:401.

*Correspondence: sarah.djabarouti@chu-bordeaux.fr

'Laboratoire de Pharmacocinétique et Pharmacie Clinique EA2968, Université Victor Segalen Bordeaux 2, Hôpital Haut-Lévêque, CHU de Bordeaux, Avenue de Magellan, 33604 Pessac Cedex, France

Full list of author information is available at the end of the article 
Table 1. Inclusion characteristics (of the 26 systemic lupus erythematosus patients) and their ability to predict clinical outcome (univariate analysis)

\begin{tabular}{|c|c|c|c|c|}
\hline Inclusion characteristic & $\begin{array}{c}\text { Total } \\
n=26\end{array}$ & $\begin{array}{c}\text { Successes } \\
n=17\end{array}$ & $\begin{array}{c}\text { Failures } \\
n=8\end{array}$ & $P$ value ${ }^{\mathrm{a}}$ \\
\hline \multicolumn{5}{|l|}{ Demographic } \\
\hline Females/Males, number & $16 / 10$ & $12 / 5$ & $4 / 4$ & $0.28^{b}$ \\
\hline Age, years & $46(35-61)^{a}$ & $46.5(35-61)$ & $46(38-61)$ & 0.9 \\
\hline Body weight, kg & $60(56-75)$ & $60(56-75)$ & $62(58-82.5)$ & 0.62 \\
\hline \multicolumn{5}{|l|}{ Disease } \\
\hline Duration before entry, months & $11(7-14)$ & $11(7-12)$ & $10(6-13)$ & 0.30 \\
\hline SLEDAl score & $0(0-2)$ & $0(0-2)$ & $0(0-2)$ & 0.20 \\
\hline$C 3, g / L$ & $1(0.6-1.1)$ & $1(0.9-1.1)$ & $0.8(0.56-0.96)$ & 0.21 \\
\hline $\mathrm{C} 4, \mathrm{~g} / \mathrm{L}$ & $0.2(0.1-0.2)$ & $0.2(0.1-0.2)$ & $0.2(0.16-0.24)$ & 0.78 \\
\hline Anti-double-stranded DNA, IU/mL & $24(6-51)$ & $8(1-32)$ & $38(9-55)$ & 0.19 \\
\hline \multicolumn{5}{|l|}{ Biological } \\
\hline GFR, mL/minute & $95(67-125)$ & $95(67-125)$ & $93.5(76-109)$ & 0.64 \\
\hline Albumin, g/L & $40(39.8-43.8)$ & $40(37.9-43.8)$ & $42.8(40-46.9)$ & 0.33 \\
\hline Aspartate aminotransferase, IU/L & $23(19-26)$ & $23(17.5-24.5)$ & $28(20-33)$ & 0.26 \\
\hline Alanine aminotransferase, IU/L & $22(15-29)$ & $19(13-24.5)$ & $25(19-37)$ & 0.21 \\
\hline Y-Glutamyltransferase, IU/L & $24(17-63)$ & $23(17-21)$ & $42(17-61)$ & 0.92 \\
\hline \multicolumn{5}{|l|}{ Treatment } \\
\hline MMF, g/day & $2(2-2)$ & $2(2-2)$ & $2(2-2)$ & 0.9 \\
\hline Corticosteroids, mg/day & $11(7-35)$ & $10(5-20)$ & $15(7-45)$ & 0.37 \\
\hline Months of MMF therapy & $2(1-3)$ & $2(1-3)$ & $2(1-3)$ & 0.56 \\
\hline Months of corticosteroids & $10(6-13)$ & $10(5-15)$ & $9(5-14)$ & 0.12 \\
\hline \multicolumn{5}{|l|}{ MPA pharmacokinetic parameters } \\
\hline$A \cup C_{0-12 h^{\prime}}$ mg.hour/L & $64.7(38.2-82)$ & $73.1(61.8-95)$ & $37.7(32-43.7)$ & 0.003 \\
\hline $\mathrm{C}_{\max } \mathrm{mg} / \mathrm{L}$ & $16.1(9.5-18.5)$ & $16.3(9.7-17.4)$ & $13.3(7-22.5)$ & 0.69 \\
\hline$T_{\text {max' }}$, hours & $1(1-2)$ & $1(1-2)$ & $1.1(1-2)$ & 0.82 \\
\hline $\mathrm{C}_{12 h^{\prime}} \mathrm{mg} / \mathrm{L}$ & $2.4(1.5-4.1)$ & $3.7(2.3-4.9)$ & $1.5(0.6-2.1)$ & 0.008 \\
\hline \multicolumn{5}{|l|}{ MPAG pharmacokinetic parameters } \\
\hline$A \cup C_{0-12 h^{\prime}} \mathrm{mg} \cdot h o u r / L$ & $775.3(475-1,026)$ & $791(635-1,166)$ & $678.8(426-840.6)$ & 0.22 \\
\hline$T_{\text {max' }}$, hours & $2(2-3)$ & $2.3(2-3)$ & $1.3(1-2)$ & 0.01 \\
\hline $\mathrm{C}_{12 h^{\prime}} \mathrm{mg} / \mathrm{L}$ & $32.1(24.3-41.9)$ & $34.7(26.4-49.2)$ & $29.8(15.5-40)$ & 0.26 \\
\hline MPAG/MPA AUC ${ }_{0-12 \mathrm{~h}}$ ratio & $11.5(8.3-20.7)$ & $10.9(6.2-14.8)$ & $18.7(14.1-22.7)$ & 0.07 \\
\hline MPAG/MPA $C_{12 h}$ ratio & $11.5(6.8-17.3)$ & $10.2(6.3-15)$ & $18.7(11.5-47.2)$ & 0.02 \\
\hline
\end{tabular}

Values are expressed as median (interquartile range) unless stated otherwise. ${ }^{a}$ Mann-Whitney $U$ test; ${ }^{b}$ Fisher exact test. AUC ${ }_{0,12}$, area under the plasma concentration-versus-time curves for hours 0 to $12 ; C_{12} h^{\prime}$ 12-hour trough concentration; $C_{\text {max' }}$ maximal concentration; GFR, glomerular filtration rate (estimated with the Cockcroft-Gault formula); MMF, mycophenolate mofetil; MPA, mycophenolic acid; MPAG, mycophenolic acid glucuronide; SLEDAl, Systemic Lupus Erythematosus Disease Activity Index; $T_{\text {max }}$ time to maximal concentration. 